\title{
Procesos colaborativos de crítica y reflexión para la coevaluación de proyectos artísticos de alumnos de Bellas Artes mediante el uso de las tecnologías móviles \\ Collaborative processes for criticism and reflection for the evaluation of students' artistic projects of Fine Arts through the use of mobile technologies
}

\author{
José Gómez Isla ${ }^{1,2}$, Felicidad García-Sánchez ${ }^{2,3}$, Juan Cruz-Benito ${ }^{3,4}$, Carmen González García ${ }^{1,2}$ \\ \{pepeisla, felicidadgsanchez, juancb, cmngonzalez\} @usal.es \\ ${ }^{1}$ Grupo de \\ Investigación Ítaca \\ Universidad de \\ Salamanca \\ Salamanca, España \\ ${ }^{2}$ Departamento de \\ Historia del Arte- \\ Bellas Artes. \\ Universidad de \\ Salamanca \\ Salamanca, España \\ ${ }^{3}$ Grupo de \\ Investigación \\ GRIAL \\ Universidad de \\ Salamanca \\ Salamanca, España \\ ${ }^{4}$ Departamento \\ de Informática y \\ Automática \\ Universidad de \\ Salamanca \\ Salamanca, \\ España
}

\begin{abstract}
Resumen- Este proyecto ha pretendido hacer partícipe a los propios alumnos de la coevaluación de proyectos artísticos y teóricos y en distintos aspectos de la enseñanza de las Bellas Artes. En estas enseñanzas se plantea una especificidad muy concreta, puesto que el grado de experimentalidad de los proyectos finales que presentan los propios alumnos y que se evalúan en las distintas asignaturas requiere de una constante labor de tutela y seguimiento por parte del docente responsable en cada una de ellas. La coevaluación, recogida en ocasiones anteriores en papel y transcrita a mano por los docentes, se ha llevado a cabo ahora mediante un Sistema de Respuesta en el Aula que garantiza el anonimato y la inmediatez en la evaluación. De esta forma el alumno, después de realizar la exposición de su proyecto, tiene una idea aproximada de lo que su trabajo sugiere y de lo que puede incluir, variar o reflexionar $\mathrm{y}$, a su vez, el resto de sus compañeros adquieren habilidades y competencias en valoraciones y críticas de proyectos artísticos contemporáneos.
\end{abstract}

Palabras clave: Coevaluación; Herramientas TIC; Bellas Artes; Sistemas de Respuesta en Aula.

Abstract- This project has sought to involve the students themselves in the co-evaluation of artistic and theoretical projects and in different aspects of the teaching Fine Arts. In this context arises very specific issues, due to the degree of experimentalism of the final projects presented by the students and that are assessed in the different subjects requires constant work of supervision and monitoring by the responsible teacher in each of them. Coevaluation, previously collected on paper and transcribed manually by teachers, has been carried out by using a Response System in the Classroom that guarantees anonymity and immediacy in the evaluation so, by this way, the student after making his presentation could have a rough idea of what its work suggests and what should be included, varied or reflected on. Also, the rest of his peers acquire by this way skills and competencies in evaluating and criticism contemporary artistic projects.

Keywords: Co-evaluation; ITC tools; Fine Arts; Classroom Response Systems.

\section{INTRODUCCIÓN}

Teniendo en cuenta que la aplicación del proyecto que se describe en este artículo reside en varias asignaturas del grado en Bellas Artes, y que la tipología docente que es recurrente en este ámbito es arquetípicamente clásica (clases prácticas en las que la forma de corregir es subjetiva y que funcionan más como un taller que como una clase magistral al uso) la incorporación de una herramienta online que permita recoger información de opinión y que ayude al docente en el sistema de evaluación puede proponer una clase participativa, activa, atenta y responsable (García-Peñalvo, 2015). Nos encontramos ante una propuesta docente innovadora e inclusiva en su concepción, ya que, como se evidencia en la literatura científica, se basa en el principio de que dotar a los alumnos de un papel de responsabilidad en el ejercicio de su aprendizaje provoca concentración e interés y otorga sentido a su presencia en el aula (García-Peñalvo, 2017).

El proyecto de innovación docente llevado a cabo durante el curso 2016-17 se ha basado en una propuesta colaborativa a través de dispositivos móviles, mediante un Sistema de Respuesta en el Aula (Carrera Escobar \& Álvarez González, 2015; Kay \& LeSage, 2009), para realizar una valoración y poner en común las reflexiones y críticas vertidas por los alumnos acerca de los proyectos artísticos presentados por sus compañeros. Esta experiencia se llevó a cabo entre alumnos de $4^{\circ}$ de grado en Bellas Artes en distintas asignaturas de la especialidad de Dibujo de la Universidad de Salamanca.

La propuesta ya se había diseñado y realizado anteriormente por el mismo equipo de profesores pero sin el uso de las tecnologías móviles. En todas las asignaturas en donde se había realizado la experiencia con anterioridad, esta propuesta de innovación se venía desarrollando hacia la mitad del curso académico para que, de ser necesario, los proyectos presentados pudiesen ser redirigidos con tiempo suficiente por 
los propios autores de los mismos, gracias a las aportaciones y comentarios de sus compañeros de curso.

Se pretendía con ello que el alumno que presentaba su proyecto artístico no sólo tomase en cuenta las valoraciones, comentarios críticos y correcciones del profesor, sino que se pretendía ampliar al máximo el espectro de colaboración mediante las sugerencias aportadas por el propio colectivo de alumnos, donde todas las impresiones y sugerencias contasen en igual medida y se sumasen a las que el profesor ya había realizado previamente mediante tutorías individualizadas. (Paredes Labra, 2013)

La dinámica llevada a cabo en ocasiones anteriores consistía fundamentalmente en que, durante el tiempo que cada alumno empleaba para presentar individualmente su proyecto ante sus compañeros, a través de la proyección de sus procesos creativos (imágenes de prueba y bocetos de su propuesta), el resto de sus compañeros escribían anónimamente en papeles en blanco sus impresiones sobre ese proyecto concreto y le hacían llegar anónimamente y con plena libertad sus valoraciones, sugerencias así como los autores y referencias bibliográficas y/o artísticas que considerasen susceptibles de consulta para ese proyecto. Tras esa exposición oral, y el feedback de sus compañeros, el alumno podía culminar con mayores garantías de éxito su proyecto a final de curso con cuantas obras finales considerase oportunas, gracias a la valoraciones motivadas que sus compañeros y el profesor habían realizado durante el proceso de gestación y plasmación del mismo.

Todas estas valoraciones eran apuntadas en una hoja sin nombre que entregaban al profesor tras finalizar la sesión en la que se exponían los proyectos. Posteriormente el profesor responsable se encargaba de transcribir y de entregar esas apreciaciones a cada alumno de forma individual. Es decir, que al alumno sólo le llegaban las valoraciones que realizaban sus compañeros sobre su propio proyecto, pero no conocía lo que ellos opinaban del resto de los proyectos presentados.

Sin embargo, con esta dinámica de trabajo surgían varias dificultades, puesto que el feedback recibido no era inmediato, ya que el alumno no recibía estas valoraciones in situ y en tiempo real durante la sesión de exposición de su trabajo, sino que debía esperar varias semanas a que dichas sugerencias fueran transcritas, reunidas y enviadas de manera individual.

Por otro lado, para el profesor de las distintas asignaturas resultaba muy laborioso tener que transcribir todas esas sugerencias, puesto que esta labor suponía un trabajo arduo y tedioso, que se sumaba al ya de por sí sobrecargado desempeño de su tarea docente.

Por todo ello, en esta ocasión se apostó por la incorporación de las aplicaciones web a través de dispositivos móviles, puesto que, hoy en día, la práctica totalidad de los alumnos universitarios disponen de teléfono móvil personal para poder incorporar sus sugerencias a través de estos dispositivos.

La herramienta utilizada en esta oportunidad la administraba la empresa Mentimeter.com (Mentimeter.com, 2017) y consistía, fundamentalmente en una arquitectura original para el diseño de una encuesta personalizada en función de necesidades del público que fuese a contestarla (en este caso el alumnado de Bellas Artes) y del tema requerido (en este caso la valoración de los proyectos artísticos presentados en clase). En esta ocasión, el diseño de la encuesta, contemplaba tanto preguntas cerradas, con escalas Likert, con varias opciones de respuesta, además de la visualización de porcentajes, como preguntas abiertas que se dejaban al final de la encuesta, para matizar algunas de las respuestas obtenidas.

\section{CONTEXTO}

En este caso, se trataba fundamentalmente de que el alumno pudiese recibir un feedback inmediato con las opiniones vertidas mediante la aplicación Mentimeter sobre su proyecto artístico. Así, tras su presentación oral, el alumno veía de forma agregada y porcentual las valoraciones y sugerencias de sus compañeros en la misma pantalla donde había presentado su proyecto.

La herramienta de diseño y administración de estas encuestas a través de dispositivos móviles resultó decisiva para que el alumno conociese en tiempo real la opinión de sus compañeros acerca de su proyecto artístico y, si algún concepto o algún proceso no había quedado suficientemente claro, se le otorgaba al alumno la oportunidad de responder a las dudas que su proyecto hubiese suscitado, o de matizar aquellos aspectos que no hubiesen sido claramente explicados. De igual forma, al finalizar la sesión, el alumno se iba ya con conocimiento de causa sobre las reacciones que su trabajo había suscitado en este primer público que lo contemplaba (es decir, sus propios compañeros).

Por supuesto, y a diferencia del sistema anterior (donde sólo el propio interesado conocía las opiniones recabadas sobre su proyecto), con este nuevo sistema, ahora sus propios compañeros de aula podían saber también de primera mano lo que el conjunto de la clase opinaba y sugería y, en cierto modo, también les iba sirviendo a ellos como elemento de comparación y aprendizaje al contrastar la visión colectiva de la clase con su propia percepción individual sobre un proyecto concreto, y averiguar así hasta qué grado existía concordancia entre la opinión general de la clase y sus propias respuestas personales.

El objetivo principal de este proyecto de innovación docente ha sido el de hacer partícipe a los propios alumnos en la coevaluación de proyectos artísticos y teóricos que han surgido a partir de la enseñanza académica en el grado de Bellas Artes, analizando transversalmente aspectos diferenciados del proceso creativo en varias asignaturas complementarias entre sí. Estas enseñanzas plantean una especificidad metodológica muy concreta, puesto que el grado de experimentalidad de los proyectos artísticos finales que presentan los alumnos y que se evalúan en las distintas asignaturas requiere de una constante labor de tutela y seguimiento por parte del docente responsable en cada una de ellas (Márquez, 2014).

Consideramos que este proyecto es sumamente innovador puesto que, en el contexto de la enseñanza superior de las Bellas Artes, se han dado pocos casos de dinámicas de coevaluación donde los alumnos se sientan también partícipes del proceso de tutela y aprendizaje a través del seguimiento colaborativo de los proyectos de sus compañeros y de las dinámicas de Sistema de Respuesta en el Aula, que agilizan esos procesos (Adams Becker et al., 2017). Así, con la puesta en común de esos proyectos y la encuesta administrada a través de dispositivos móviles, no se delega toda la 
responsabilidad del proceso de enseñanza-aprendizaje en la figura del profesor responsable de cada asignatura. Ya sea por su experiencia adquirida como docente o por su bagaje personal, no en todas las ocasiones el profesor puede situarse en una posición cercana a la problemática particular que se les plantea a algunos alumnos. Por falta de conocimiento del contexto en el que se enmarcan algunos proyectos, el docente a veces ignora las fuentes de referencia o las motivaciones creativas que han impulsado a desarrollar un proyecto concreto de la forma en la que lo hacen.

Además del objetivo principal, ya descrito más arriba, los objetivos específicos que este proyecto de innovación técnica ha pretendido alcanzar son los siguientes:

1. Fomentar la participación activa del estudiante y su implicación en tareas de crítica y reflexión en torno a los proyectos artísticos y teóricos de sus compañeros de asignatura.

2. Fomentar la implicación en el aula mediante el uso de las TIC en determinadas propuestas de coevaluación entre el alumnado de forma agregada, garantizando así el anonimato que confieren estas aplicaciones web para que sus respuestas y opiniones sean libres, sinceras y para que sirvan con utilidad y eficacia en el análisis constructivo y colaborativo de los proyectos presentados.

3. Desarrollar en el estudiante (tanto de forma individual como colectiva) un espíritu crítico para analizar los proyectos presentados. Este análisis no sólo se centraría en la crítica constructiva y la valoración de los proyectos ajenos, sino también en un proceso de reflexión y autoevaluación del propio proyecto artístico en cuantas asignaturas teóricoprácticas se vean afectadas por la implementación de este proyecto de innovación.

4. Establecer criterios colegiados entre distintas áreas de conocimiento del Grado en Bellas Artes y entre distintas materias teórico-prácticas que permitan mejorar la coordinación entre cursos y materias de esta titulación universitaria.

5. Fomentar la comprensión de los mecanismos y aspectos del arte que generan procesos de creación complejos.

6. Fomentar entre los alumnos su capacidad de análisis y criterios para comparar, relacionar y reflexionar sobre sus proyectos artísticos personales en el contexto creativo del aula.

7. Fomentar la exposición oral de sus propuestas artísticas para mejorar su competencia comunicativa en lo referente a sus propios procesos de creación.

8. Fomentar la discusión crítica personal y agregada, así como el debate reflexivo entre alumnos a través del uso de aplicaciones web.

9. Incentivar al alumno para mejorar sus proyectos creativos mediante la motivación obtenida en los borradores expuestos por sus propios compañeros a través de las sesiones participativas de puesta en común.

10. Incentivar las habilidades comunicativas del alumno para difundir adecuadamente por medio de las TIC sus proyectos artísticos dirigidos al público interesado.

11. Fomentar el trabajo colaborativo de análisis y evaluación de proyectos artísticos mediante estrategias de interacción web.

12. Hacer extensivas entre los alumnos las tareas de coevaluación, argumentación crítica y reflexión al ámbito del arte contemporáneo (Pastor, Pascual, \& Martín, 2005).

Como hemos dicho anteriormente, el público objetivo de esta propuesta de innovación han sido los propios alumnos de Bellas Artes, en su último año de grado universitario. Para ello se les ha propuesto previamente la realización de un trabajo artístico para que pongan en marcha muchos de los mecanismos del proceso creador, que van desde su ideación, pasando por la puesta en marcha de bosquejos y propuestas a partir de una temática elegida, los conceptos plasmados materialmente en forma de imágenes, así como la confección de cuadernos de artista, bocetos, borradores, para acabar con el desarrollo de una metodología de trabajo y la generación de un estilo, poética personal o lenguaje artístico específico que les defina en su último año de carrera como creadores consumados (Paredes Labra, 2013).

\section{DESCRIPCIÓN}

El plan de trabajo para esta dinámica de innovación docente se desglosó en varias fases, personalizándolo, eso sí, para todas aquellas asignaturas en las que se implementó dicha propuesta:

En primer lugar, fue necesario diseñar un modelo de cuestionario ad hoc para cada una de las asignaturas, puesto que los procesos de enseñanza-aprendizaje y metodologías de trabajo variaban sensiblemente entre las distintas asignaturas. Los cuestionarios fueron confeccionados concienzudamente con diversas preguntas destinadas a conocer el grado de consecución de objetivos que cada alumno encuestado considerase que había alcanzado (o que debería alcanzar) cada uno de los proyectos presentados en el aula. A continuación, se proporcionan, como ejemplo, las preguntas incluidas en el cuestionario de coevaluación entre alumnos dentro de las asignaturas de Dibujo del $4^{\circ}$ curso del Grado en Bellas Artes:

1. ¿Consideras adecuado el grado de experimentación e innovación (del proyecto)? En esta pregunta, los estudiantes evalúan los siguientes aspectos mediante una escala Likert 1-5 (1-nada, 5mucho):

a. Grado de experimentación técnica

b. Grado de innovación

2. ¿Consideras que el proyecto ha alcanzado un estilo maduro? En esta pregunta los alumnos podían elegir entre tres opciones:
a. Sí
b. No
c. Está muy verde / Necesito ver más

3. ¿Crees que es un proyecto bien logrado conceptual y técnicamente? En esta pregunta los alumnos podían elegir tres opciones:

a. Sí

b. No, aún no 
c. No lo sé. Necesito verlo terminado

4. ¿Está bien documentado y explicado el proyecto: antecedentes, procesos, resultados? Igual que en la primera pregunta, en esta ocasión los alumnos puntúan cada uno de los aspectos mediante una escala Likert entre 1 (nada) y 5 (mucho). Los aspectos a valorar fueron:
a. Antecedentes
b. Procesos
c. Resultados

5. Haz alguna sugerencia para mejorar este proyecto. Pregunta de respuesta libre (en modo texto)

Una vez diseñado el cuestionario, se celebró una sesión específica para incorporarla a la dinámica de la asignatura como metodología de trabajo colaborativo, lo que fomentaba la participación de forma interactiva y online en el proceso de evaluación de los proyectos a través de la aplicación web Mentimeter (Mentimeter.com, 2017).

Antes de llevar a cabo dicha sesión se realizó una evaluación pre-test de la herramienta mediante un focus group entre profesores de las asignaturas para comprobar su eficacia y rapidez tanto en la captación como en la recogida de las respuestas y exposición de los resultados y estadísticas de la misma en forma de gráficos.

Se realizó de igual modo, como fase de prueba, un ensayo de manejo de la herramienta con los alumnos, destinado a familiarizarse en el uso de aplicación en el aula a través del móvil. Dicha minisesión de entrenamiento con la herramienta Mentimeter tuvo lugar una semana antes de la exposición de los proyectos, de forma que tanto profesores como alumnos pudieran comprobar en tiempo real el grado de facilidad/dificultad de manejo de la aplicación para obtener los resultados esperados de forma interactiva.

Se llevó a cabo la sesión colectiva por cada asignatura en la que se realizó la exposición oral de cada uno de los proyectos en el aula. Y en esta misma sesión se administró la encuesta a partir de la aplicación online Mentimeter para que fuese respondida individualmente por cada alumno con su dispositivo móvil. En cualquier caso, los resultados (que aparecían en la pantalla de proyección del aula en tiempo real) se mostraban de forma estadística y agregada. De este modo la coevaluación de los proyectos presentados por cada alumno se podía ver mediante la suma de valoraciones y propuestas, por lo que podemos decir que las sugerencias y valoración global siempre se produjo de forma colaborativa entre todos los miembros que integraban la clase. El uso de la aplicación conllevaba un tiempo mínimo de reflexión y respuesta para que los alumnos pudiesen completar las preguntas sobre el grado de adecuación del proyecto respecto a los objetivos propuestos, así como el nivel de calidad, coherencia y madurez que ellos consideraban que habían alcanzado cada uno de los proyectos presentados.

En una sesión de tutoría posterior se discutieron con cada alumno de forma individualizada las valoraciones, sugerencias y propuestas de mejora que había recibido de sus compañeros en la sesión de coevaluación de su proyecto. Previamente se le habían enviado a cada alumno por correo electrónico los pantallazos con los gráficos de respuesta así como la hoja Excel que recogía tanto las respuestas abiertas con las sugerencias cualitativas vertidas como las estadísticas y porcentajes generados a partir de las respuestas a las preguntas cerradas que había obtenido su proyecto.

Finalmente se realizó una sesión post-test con los profesores para valorar la eficacia y utilidad del modelo de encuesta diseñado al efecto para cada una de las asignaturas, así como una propuesta de modificación de aquellas preguntas que funcionaron peor y sustituirlas, o directamente eliminarlas, del cuestionario para la siguiente ocasión. Del mismo modo, se realizó otra sesión final con los alumnos en la que estos proporcionaron su opinión sobre el proceso de coevaluación: pertinencia, utilidad, satisfacción, etc.

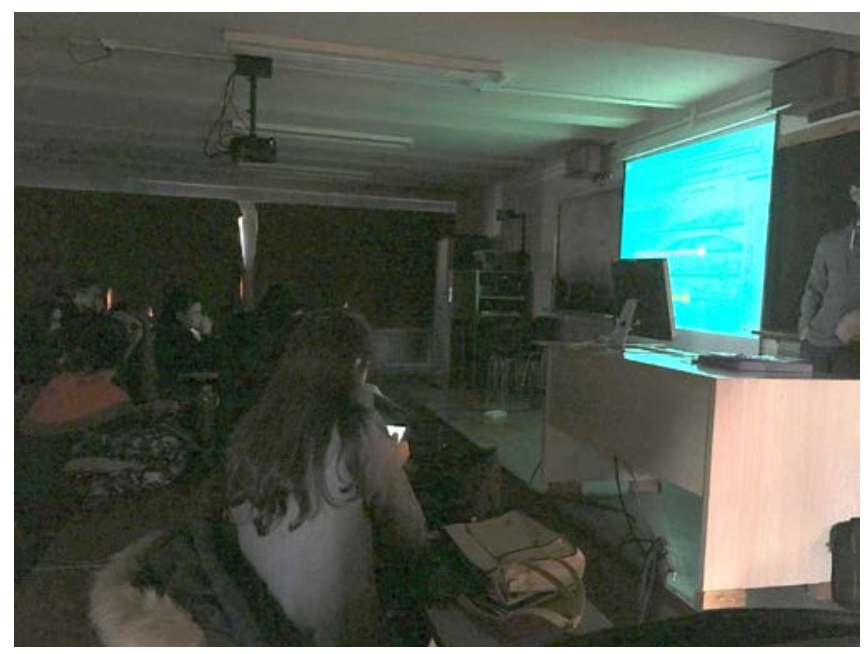

Figura 1. Una de las sesiones en las que se utilizó la herramienta.

\section{RESULTADOS}

Una vez administrada la encuesta, se obtuvieron los resultados colectivos que, de algún modo, reflejaban la opinión general de los alumnos de la asignatura con respecto a un determinado proyecto. La propuesta de innovación docente se consideró exitosa puesto que entre el alumnado se administró una segunda encuesta sobre el grado de satisfacción obtenido personalmente mediante esta dinámica de coevaluación de sus proyectos. Los resultados de esta segunda encuesta obtuvieron una valoración significativamente positiva, lo que respondía a las expectativas planteadas, así como al grado de utilidad que los alumnos consideraron que tenía esta dinámica grupal.

Entre las preguntas propuestas en esta encuesta de evaluación se encuentran las siguientes:

1. ¿Crees adecuado el tiempo asignado (5 ó 10 minutos) para exponer tu proyecto? Se plantean diversas respuestas: suficiente, insuficiente, corto, adecuado

2. ¿Crees adecuado el tiempo total empleado en la sesión? Se plantean diversas posibles respuestas: adecuado, excesivo, se debería acortar.

3. ¿Consideras útil haber podido ver el proceso de trabajo de tus compañeros?: Utilidad. Respuesta en una escala Likert 1-5 (1 nada, 5 mucho) 
4. ¿Consideras útiles las opiniones de tus compañeros para mejorar tu proyecto y/o para aclarar ideas? Distintas posibles respuestas: útil, útil para otros compañeros y no para mí, no.

5. ¿Consideras que a esta altura del curso ya tienes suficientemente bien encauzado tu proyecto? Respuestas disponibles:

o Sí, lo tengo claro y empiezo a obtener resultados definitivos

o Estoy en proceso de resolución aunque el proyecto aún no esté del todo definido

o No, necesito aún más tiempo para madurar el proyecto

6. Indica tu grado de satisfacción en lo relativo a la dinámica de esta sesión: Satisfacción. Respuestas en una escala Likert 1-5 (1 nada, 5 mucho).

7. Si crees que la encuesta anterior puede mejorarse o quieres sugerir preguntas más pertinentes, por favor hazlo. Pregunta de respuesta abierta.

En consecuencia de lo presentado anteriormente, con esta herramienta metodológica para coevaluar procesos artísticos, diseñada ex profeso para pulsar la opinión del alumnado sobre los proyectos presentados, no sólo el interesado recibía la crítica constructiva sobre fortalezas y debilidades (virtudes y defectos) que sus propios compañeros habían detectado en su proyecto (Figura 2, ejemplo del feedback recibido en la pregunta 1 del cuestionario de coevaluación); también el resto de sus compañeros que respondían a la encuesta veían los resultados y percibían así una idea general del sentir mayoritario de toda la clase sobre un determinado proyecto. De este modo podían comparar de forma inmediata su grado de acuerdo o desacuerdo con la opinión generalizada del grupo y contrastar así su capacidad crítica con la del resto de sus compañeros de asignatura. El objetivo final consistía en que todos aprendiesen de esta dinámica de grupo, y no sólo el alumno que había expuesto su proyecto personal. Al contrario, todos debían conocer las críticas del resto de la clase, así como los argumentos y las razones por las cuales un proyecto les parecía más o menos acertado, y dónde detectaban los puntos débiles del mismo que podrían ser susceptibles de mejora.

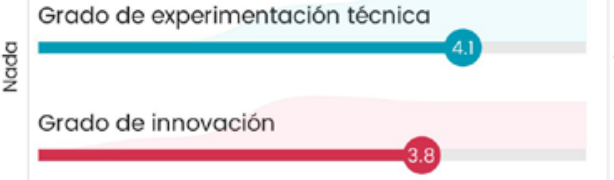

Figura 2: Ejemplo de resultados que recibe un alumno sobre la primera pregunta del cuestionario de coevaluación en relación a su proyecto presentado.

De igual modo, la dinámica de este proyecto de innovación docente sirvió también para que el profesor de cada asignatura pudiese contrastar y comparar los resultados de sus propias correcciones, sugerencias y valoraciones de cada proyecto respecto a la opinión mayoritaria del resto de la clase. Fue muy revelador comprobar cómo, en líneas generales, coincidían punto por punto y en casi todos los proyectos ambas evaluaciones (la del profesor y la global de los alumnos)

En general, la opinión recabada mediante entrevistas por parte de los distintos profesores involucrados ha sido positiva o muy positiva. Respecto a la satisfacción de los alumnos, tomando los resultados de la encuesta de satisfacción sobre la actividad de coevaluación (preguntas 3, 4, 6 de la encuesta presentada en esta sección), se puede afirmar que los resultados son muy positivos. Concretamente, para la pregunta ¿Consideras útil haber podido ver el proceso de trabajo de tus compañeros?, la valoración recogida es de 4,36 sobre un máximo posible de 5 en la escala propuesta (desviación típica 0,995). Por otro lado, para la pregunta ¿Consideras útiles las opiniones de tus compañeros para mejorar tu proyecto $y / 0$ para aclarar ideas?, la opinión de los alumnos ha sido mayoritariamente positiva: 18 de las 25 respuestas (72\% del total) se corresponden con la opción más favorable: "Sí, me resulta muy útil”, mientras que las otras 7 se corresponden con la opción "Aunque a mí personalmente no me ha servido, sí veo que pueda ser útil para otros” y no se recoge ninguna respuesta negativa. En último lugar, respecto a la pregunta 6 de la encuesta Indica tu grado de satisfacción en lo relativo a la dinámica de esta sesión: Satisfacción, se vuelven obtenerse resultados bastante positivos. La valoración media de las respuestas obtenidas es de 3,96/5 (desviación típica: 1,21). Con estos datos, se puede concluir que la experiencia ha sido positiva también a nivel de alumnos. En relación a la pregunta 7, sobre qué aspectos mejorarían, cabe destacar que las mejoras principales se refieren al ritmo de la clase (más descansos, decrementar el número de proyectos presentados por sesión, etc.) más que a ninguna crítica relacionada con la discusión de los proyectos y su coevaluación mediante los métodos presentados en este proyecto de innovación.

\section{CONCLUSIONES}

En líneas generales, y en relación a las opiniones de los alumnos, sus calificaciones y la comparación de estas con los resultados obtenidos en cursos anteriores, se constató la obtención de un mejor rendimiento en el proceso de enseñanza-aprendizaje del proceso creativo por parte del alumnado, así como del desarrollo de una mejor capacidad crítica para discriminar los aspectos conceptuales, creativos, técnicos y formales de cada proyecto a la hora de valorarlos por separado y de detectar las virtudes y las posibles deficiencias de cada proyecto analizado.

De acuerdo a lo observado por los docentes, y a las opiniones expresadas por los alumnos mediante una encuesta de satisfacción presentada al final de la asignatura, se puede decir que el proyecto de innovación docente ha podido contribuir en los siguientes aspectos:

1. Mejorar la capacidad de los alumnos para identificar $\mathrm{y}$ entender los problemas del arte y establecer y discriminar los distintos procesos del arte que potencian el desarrollo de la creatividad y la suficiencia técnica a la hora de dibujar y plasmar visualmente esa creatividad en forma de proyecto. 
2. Mejorar la capacidad para comprender y valorar discursos artísticos en relación con la propia obra. Se lograron establecer así medios de comparación para relacionar la obra artística personal con el contexto creativo global de la asignatura.

3. Mejorar las capacidades del alumno para exponer oralmente y por escrito con claridad las fases, procesos y problemas artísticos complejos y sus propios proyectos creativos.

4. Mejorar la capacidad del alumno para identificar los problemas artísticos y las influencias socio-culturales del contexto actual que hacen posible unos discursos artísticos determinados, así como identificar los condicionantes que inciden en la creación artística y analizar las diversas estrategias de producción creativa en el ámbito de las Bellas Artes.

5. Mejor desarrollo de la capacidad crítica y autorreflexiva del alumno para valorar y evaluar las fortalezas y debilidades de los proyectos artísticos propios y ajenos. Indudablemente se logró mejorar su capacidad reflexiva en torno a las cualidades que debe tener una propuesta artística sólida.

6. Mejorar significativamente el grado de participación de los alumnos en clase en lo referente a la crítica y valoración de proyectos artísticos, fomentando la discusión colectiva y constructiva entre ellos mediante las respuestas sinceras que permitía el formato anónimo de la encuesta a través de la aplicación web y el posterior debate de los resultados.

7. Mejorar también la percepción de la capacidad individual de cada alumno para abordar proyectos de investigación artística a raíz de los ejemplos aportados por sus propios compañeros en la exposición oral de sus respectivas propuestas de creación. Esta puesta en común les permitió abordar el final de su proyecto con una mayor seguridad gracias al refrendo y las sugerencias de mejora aportadas de forma constructiva por sus compañeros.

8. Mejorar significativamente las habilidades comunicativas de los alumnos para expresar, tanto verbalmente como por escrito, y a través de las TIC (mediante presentaciones en powepoint, prezi, etc), sus propias propuestas y difundir de la mejor manera sus propios proyectos artísticos. De igual modo, se consiguieron mejorar las habilidades expresivas del alumno para saber traducir verbalmente las propias ideas artísticas y poder transmitirlas adecuadamente (Dominguez \& Vazquez, 2017).

9. Se consideró muy positiva la propuesta de innovación docente para introducir a los alumnos en tareas de coevaluación grupal, argumentación crítica y reflexión sobre las propuestas presentadas en el marco del arte contemporáneo a través de los ejemplos cercanos de procesos creativos aportados por sus propios compañeros.

10. Por último, se incentivó a los alumnos para que, a partir de esta dinámica, se animaran a generar ellos mismos sus propias propuestas colaborativas a través de las TIC (Cabero, 2002).

\section{AgRAdECIMIENTOS}

Queremos expresar nuestro agradecimiento al programa de mejora de la calidad docente promovido por el Vicerrectorado de Docencia de la Universidad de Salamanca que, a través de la convocatoria de ayudas a proyectos de innovación y mejora docente, financió el presente proyecto ID2016/096 e hizo posible que tanto la metodología, como la difusión de los resultados se pudieran llevar a efecto mediante dicha financiación. Felicidad García-Sánchez agradece, a su vez, a la Universidad de Salamanca y al Banco Santander la financiación de su contrato pre-doctoral como investigadora en formación. Por su parte, Juan Cruz-Benito agradece al Fondo Social Europeo y a la Consejería de Educación de la Junta de Castilla y León la financiación de su contrato predoctoral.

\section{REFERENCIAS}

Adams Becker, S., Cummins, M., Davis, A., Freeman, A., Hall Giesinger, C., \& Ananthanarayanan, V. (2017). NMC horizon report: 2017 higher education edition. Austin, Texas: The New Media Consortium.

Cabero, J. (2002). Las TICs en la universidad. Sevilla, MAD.

Carrera Escobar, D., \& Álvarez González, L. (2015). Sistemas de Respuesta en Aula de Libre Distribución para uso con Dispositivos Móviles. Paper presented at the $\mathrm{V}$ Encuentro. Conferencias Chilenas en Tecnologías del Aprendizaje., Arica.

Dominguez, H. L., \& Vazquez, H. C. (2017). El uso de las TIC y sus implicaciones en el rendimiento de los alumnos de bachillerato. Un primer acercamiento. Teoría de la Educación.Educación y Cultura en la Sociedad de la Información, 18(1), 21-38.

García-Peñalvo, F. J. (2015). Mapa de tendencias en Innovación Educativa. Teoría de la educación. educación y cultura en la sociedad de la información, 16(4), 6-23.

García-Peñalvo, F. J. (2017). Tendencias en las posibilidades tecnológicas del eLearning.

Kay, R. H., \& LeSage, A. (2009). Examining the benefits and challenges of using audience response systems: A review of the literature. Computers \& Education, 53(3), 819827.

Márquez, M. d. C. B. (2014). La evaluación por competencias en la docencia universitaria del Grado en Bellas Artes. Historia y Comunicación Social, 18, 865-877.

Mentimeter.com. (2017). Website. Retrieved from https://www.mentimeter.com/

Paredes Labra, J. (2013). Docentes noveles universitarios y su enseñanza con TIC.

Pastor, V. L., Pascual, M. G., \& Martín, J. B. (2005). La participación del alumnado en la evaluación: la autoevaluación, la coevaluación y la evaluación compartida. Rev. Tándem Didáctica Educ. Fís., 17, 2137. 